\title{
O uso de cenários cotidianos baseados na ferramenta educacional My World com mães de crianças e adolescentes com deficiência auditiva
}

\section{The use of everyday scenarios based on the educational tool "My World" in mothers of children and adolescents with hearing loss}

\author{
Mariani da Costa Ribas do Prado', Dagma Venturini Marques Abramides²
}

\begin{abstract}
RESUMO
Objetivo: Identificar a concepção dos participantes sobre o uso dos cenários e personagens da ferramenta My World para o treinamento de habilidades sociais educativas cotidianas com mães de crianças e adolescentes com deficiência auditiva. Métodos: Estudo exploratório sobre a análise dos relatos dos participantes quanto ao uso dos cenários e personagens da ferramenta para desenvolvimento de práticas parentais positivas. O estudo foi desenvolvido em uma clínica-escola de Fonoaudiologia de uma faculdade do interior do estado de São Paulo, Brasil, com três mães de crianças com deficiência auditiva, que realizavam terapia fonoaudiológica naquele local. Para coleta dos dados, foi aplicado um questionário sobre os elementos da ferramenta. Resultados: Observou-se que os elementos da ferramenta parecem ter contribuído para que as mães identificassem mais concretamente dificuldades e facilidades na interação com seus filhos com deficiência auditiva. Conclusão: Acredita-se que este estudo possa auxiliar na implantação de ferramentas que favoreçam a participação dos familiares no processo de reabilitação auditiva, contribuindo, assim, para maior probabilidade de sucesso terapêutico.
\end{abstract}

Palavras-chave: Deficiência auditiva; Reabilitação; Criança; Adolescente; Família

\begin{abstract}
Purpose: To identify the participants'opinion about the use of scenarios and characters of the educational tool "My World", in parents of children with hearing loss. Methods: This exploratory study analyzed the reports of participants about utilization of the tool's scenarios and characters for the development of positive parental practices. The study was conducted at a university speech-language pathology clinic in a city at the countryside of the state of Sao Paulo, Brazil, on three mothers of children and adolescents with hearing loss, who received speech therapy at the clinic. Data were collected using a questionnaire about the tool elements. Results: The tool elements seemed to contribute for the mothers to more precisely identify the ups and downs in the interaction with their children with hearing loss. Conclusion: This study can aid the development of tools to favor the participation of family members in the auditory rehabilitation of their children, thus contributing to increase possibility of therapeutic success.
\end{abstract}

Keywords: Hearing loss; Rehabilitation; Child; Adolescents; Family

\footnotetext{
Trabalho realizado na Faculdade de Odontologia de Bauru - FOB, Universidade de São Paulo - USP - Bauru (SP), Brasil.

${ }^{1}$ Hospital de Reabilitação de Anomalias Craniofaciais - HRAC, Universidade de São Paulo - USP - Bauru (SP), Brasil.

${ }^{2}$ Departamento de Fonoaudiologia, Faculdade de Odontologia de Bauru - FOB, Universidade de São Paulo - USP - Bauru (SP), Brasil.

Conflito de interesses: Declara-se que, para execução deste estudo, bem como para sua publicação, não houve e não há qualquer conflito de interesse por parte das autoras.

Contribuição dos autores: MCRP desenvolveu o estudo relatado neste artigo como parte de sua tese de doutorado; DVMA orientadora, realizou orientações pertinentes para execução do presente trabalho.

Financiamento: Nada a declarar.

Autor correspondente: Mariani da Costa Ribas do Prado. E-mail: mprado@usp.br

Recebido: Outubro 16, 2017; Aceito: Julho 10, 2018
} 


\section{INTRODUÇÃO}

Desde a década de 80 , vem sendo proposta uma mudança paradigmática, em relação aos modelos de intervenção precoce na área da deficiência auditiva, preconizando as práticas centradas na família como aquelas que promovem melhores resultados para o desenvolvimento da criança ${ }^{(1)}$. Neste sentido, foi realizado um estudo que procurou conhecer e descrever as características das atividades cotidianas que contribuem para o desenvolvimento e melhoria da comunicação e da linguagem, como forma de contribuir para o desenvolvimento de práticas centradas na família. Para isto, foram utilizados dois instrumentos, sendo duas variações da La Escala de Valoración de la Enseñanza de la Lengua Oral en contexto Familiar - EVALOF, que avaliou a percepção dos pais sobre sua interação com os filhos e utilizou uma entrevista para apreender as atividades diárias de quatro famílias com crianças de 5 anos de idade. Para avaliação da linguagem das crianças, foi utilizado a Prova de Linguagem Oral de Navarra-PLON. As pesquisadoras do estudo verificaram que todas as crianças precisavam melhorar seu desenvolvimento linguístico e que os pais poderiam usar, com mais frequência, as rotinas diárias para conversar com os filhos. Desta forma, ressaltaram a necessidade de que o cotidiano de cada família seja o ponto de partida das intervenções centradas nas famílias, visando promover alterações que sejam incorporadas ao seu dia a dia ${ }^{(2)}$.

Na área da Audiologia, estudos têm demonstrado a necessidade de que terapias fonoaudiológicas sejam desenvolvidas de acordo com o paradigma das práticas centradas nas famílias ${ }^{(3-5)}$. Desta forma, a reabilitação auditiva vem passando por mudanças em sua estrutura, enfatizando-se, cada vez mais, a importância da família nesse processo, visto que o sistema familiar é primordial para a construção da linguagem na criança ${ }^{(6)}$. As crianças com deficiência auditiva que apresentam os melhores desempenhos na terapia fonoaudiológica, em termos de desenvolvimento da linguagem oral, são aquelas nas quais se observa a continuidade do trabalho terapêutico no ambiente doméstico ${ }^{(7)}$.

Frente a este cenário, em 2012, foi realizada uma conferência na Áustria, com o objetivo de se discutir sobre a sistematização das intervenções precoces centradas na família, na área da deficiência auditiva. Na conferência, foram estabelecidos os seguintes princípios norteadores para as práticas centradas nas famílias: 1 - Acesso precoce, ágil e equitativo aos serviços; 2 - Parcerias entre familiares e prestadores de serviço; 3 - Escolhas e decisões conscientes; 4 - Suporte social e emocional às famílias; 5 - Promoção da interação pais/crianças; 6 - Uso de tecnologias assistivas e suporte à comunicação; 7 - Profissionais qualificados; 8 - Trabalho em equipe; 9 - Monitoramento do progresso; 10 - Programas monitorados ${ }^{(8)}$.

Em revisão de literatura, buscou-se descrever como e porque as práticas centradas na família se constituem fundamentais para o desenvolvimento da comunicação em crianças com deficiência auditiva. As autoras ressaltaram que a principal mudança desse tipo de prática se relaciona à alteração do foco da intervenção fonoaudiológica da criança, individualmente, para o apoio e à capacitação de seus parceiros de comunicação. A partir dos estudos analisados, concluíram que as crianças com maior sucesso terapêutico são aquelas nas quais se constatam as interações cotidianas incorporadas aos atendimentos ${ }^{(4)}$.
Por outro lado, foi realizada uma pesquisa que apontou que existe dificuldade para implantação de práticas centradas na família em conformidade com todos os princípios norteadores estabelecidos. Os autores investigaram, por meio de entrevista semiestruturada, as informações recebidas em programas de intervenção precoce centrados na família, por 12 pais ouvintes de crianças com deficiência auditiva, sendo 11 mães e um pai. Por meio da análise temática dos relatos das entrevistas, os autores verificaram que os programas estão seguindo parcialmente os princípios norteadores das práticas de intervenção precoce centradas na família, pois, segundo os participantes, apesar dos profissionais orientarem sobre a importância da interação durante as atividades diárias para o desenvolvimento das habilidades linguísticas da criança, existe carência de orientações individualizadas e específicas à realidade de cada criança ${ }^{(3)}$.

Em estudo sobre interação de pais ouvintes com seus filhos com deficiência auditiva, ressaltou-se a importância do enfoque familiar para o sucesso do desenvolvimento da comunicação oral na terapia fonoaudiológica. Foi realizada a análise de 18 filmagens, por meio da Escala de Índice de Comportamentos, que promove a comunicação, com nove pais de crianças com deficiência auditiva e nove pais de adolescentes, também com deficiência auditiva. Como resultados, verificou-se que os pais que frequentavam um programa cujo objetivo era o desenvolvimento da função auditiva e da comunicação oral demonstraram estratégias de comunicação adequadas frente a uma situação controlada. No entanto, para as pesquisadoras, esses dados não permitiram afirmar se tais atitudes também estão presentes em situações cotidianas. Diante disso, enfatizaram a necessidade de estudos e de estratégias que possibilitem a generalização dessas habilidades ${ }^{(5)}$.

Tem sido ressaltada a utilização insuficiente dos cenários de atividades diárias das crianças e seus familiares, com reflexos negativos para o aproveitamento eficaz das oportunidades de aprendizagem infantil ${ }^{(9)}$. Uma organização dinamarquesa de profissionais sem fins lucrativos, denominada IDA Institute, tem preconizado o uso de ferramentas durante o processo de reabilitação auditiva, destacando-se a My World, por contemplar a utilização desses cenários de atividade diária, durante esse processo. Trata-se de uma ferramenta que tem, como proposta, a compreensão da perda auditiva a partir do ponto de vista da pessoa com deficiência auditiva, por meio da manipulação de seus componentes (cenários, personagens e objetos). Tal ferramenta vem sendo utilizada com sucesso na clientela infantojuvenil que frequenta a clínica-escola de Fonoaudiologia de uma cidade do interior do estado de São Paulo.

A proposta para este estudo foi a utilização dos cenários e personagens da ferramenta My World com mães de crianças e adolescentes com deficiência auditiva, com o intuito de que, de maneira mais concreta e numa linguagem mais simplificada, pudessem manifestar e aprimorar as habilidades parentais em diferentes situações, a partir da manipulação de seus componentes, criando-se as cenas do dia a dia para descrever a comunicação entre as díades pai/mãe-filho. Sendo assim, o objetivo desta pesquisa foi identificar a concepção dos participantes sobre o uso dos cenários e personagens da ferramenta My World para o treinamento de habilidades sociais educativas cotidianas com mães de crianças e adolescentes com deficiência auditiva. 


\section{MÉTODOS}

Trata-se de uma pesquisa qualitativa exploratória sobre as concepções das mães quanto ao uso da ferramenta My World para treinamento de habilidades sociais educativas cotidianas. Este estudo se constituiu como a primeira etapa do projeto aprovado em sua íntegra pelo Comitê de Ética em Pesquisa com Seres Humanos (número do parecer e data da relatoria: 918.578, 09/12/2014), conforme Resolução 466/12. Foi desenvolvido em uma clínica-escola de Fonoaudiologia de uma cidade do interior do estado de São Paulo. Na ocasião da coleta de dados, havia 20 pacientes em tratamento na área da Audiologia e todos os responsáveis foram convidados, por meio de carta convite, a participarem da pesquisa. Três mães compareceram ao encontro, sendo denominadas M1, M2 e M3 e seus filhos com deficiência auditiva, respectivamente, P1, P2 e P3. Os dados sociodemográficos dos participantes encontram-se destacados nos Quadros 1 e 2.

As mães participantes tinham entre 39 e 63 anos (média de idade de 48 anos), sendo que uma atuava profissionalmente e tinha ensino superior completo. As demais eram donas de casa e tinham, respectivamente, ensino médio completo e ensino fundamental incompleto. A classificação socioeconômica variou de baixa inferior a média inferior. As crianças eram dois meninos e uma menina, com idades entre 10 e 11 anos, frequentando o $5^{\circ}$ ano escolar. Dois deles tinham perda auditiva profunda bilateral e usavam implante coclear e o outro tinha perda moderada e usava AASI. As crianças tinham de um a dois irmãos (Quadro 2).

Os dados sociodemográficos, tanto das mães, como dos pacientes, foram extraídos de seus prontuários,além das informações sobre a deficiência auditiva. A classificação socioeconômica considerou renda familiar, escolaridade, ocupação, número de pessoas na casa e tipo de habitação ${ }^{(10)}$.

A ferramenta My World foi criada pelos profissionais do IDA Institute ${ }^{(11)}$, os quais criam e compartilham conhecimentos inovadores, para ajudar os profissionais de saúde auditiva a enfrentar os desafios psicológicos e sociais da perda auditiva e aplicar métodos de cuidados centrados no paciente. A ferramenta está disponível no site para download gratuito e as instruções em Português foram realizadas por um grupo de fonoaudiólogos brasileiros.
O instrumento My World auxilia na compreensão da perda auditiva, a partir do ponto de vista da pessoa com deficiência auditiva. Seus cenários consistem em três ambientes diferentes (uma sala de aula, uma casa e uma área ao ar livre), personagens que aludem à família, escola e amigos, objetos que remetem ao cotidiano e cartões com ícones que expressam sentimentos positivos e negativos. $\mathrm{O}$ indivíduo pode colocar figuras móveis em vários ambientes, para descrever os sucessos e os desafios de comunicação do dia a dia. Ao jogar com os componentes da ferramenta, pode-se exteriorizar a perda auditiva e discutir como o indivíduo se comunica com os outros, de uma forma concreta e não ameaçadora. Sendo assim, a ferramenta fornece informações sobre os padrões de comunicação da pessoa com deficiência auditiva, reforçando padrões positivos e formulando estratégias para lidar com todos os desafios do dia a dia.

Para avaliação da pertinência do uso dos cenários e personagens da ferramenta, foi utilizado um questionário com as seguintes perguntas: $\mathrm{O}$ que o(a) senhor(a) achou dos cenários e personagens?; A utilização dos cenários e dos personagens, de alguma forma, pode ajudar a refletir sobre a relação pais-filhos?; O que o(a) senhor(a) mudaria e/ou acrescentaria nesses cenários e personagens?.

A utilização dos cenários e personagens da ferramenta educacional My World se desenvolveu em sete etapas: 1 - Contato com os profissionais do IDA Institute, para autorização do uso de seus cenários e personagens; 2 - Estabelecimento de parceria com o setor de tecnologia educacional da faculdade onde foi desenvolvido o trabalho, para o redesenho dos elementos da ferramenta; 3 - Apresentação do projeto, com ênfase no uso da ferramenta, aos grupos de estagiários de Fonoaudiologia que realizavam a terapia de crianças e adolescentes com deficiência auditiva; 4 - Distribuição, pelas estagiárias, de uma carta-convite às mães das crianças; 5 - Assinatura do convite pelas mães; 6 - Apresentação às mães dos diferentes ambientes, personagens $\mathrm{e}$ figuras contidos na ferramenta; 7 - Preenchimento do questionário referente à opinião das mães sobre o uso de tal ferramenta.

As etapas 5 e 6 se desenvolveram com as mães que compareceram ao encontro previamente agendado, que foi realizado em horário diferente da terapia fonoaudiológica do filho com deficiência auditiva. Todas as mães, independente da faixa etária do(a) filho(a) e do agendamento da fonoterapia, foram convidadas. Na ocasião do encontro, 20 pacientes (crianças e adolescentes) com deficiência auditiva estavam inseridos na

Quadro 1. Características sociodemográficas das mães participantes

\begin{tabular}{|c|c|c|c|c|c|c|}
\hline Participante & Idade & Profissão & $\begin{array}{c}\text { Estado } \\
\text { Civil }\end{array}$ & Escolaridade & Renda Familiar & $\begin{array}{c}\text { Classificação } \\
\text { Socioeconômica }\end{array}$ \\
\hline M1 & 42 anos & Professora Universitária & Casada & Ensino superior & Acima de 6 salários mínimos & Média inferior \\
\hline M2 & 39 anos & Do lar & Casada & Ensino médio & 4 salários mínimos & Baixa superior \\
\hline M3 & 63 anos & Do lar & Casada & $\begin{array}{c}\text { Ensino fundamental } \\
\text { incompleto }\end{array}$ & 3 salários mínimos & Baixa inferior \\
\hline
\end{tabular}

Legenda: $M$ = Mãe

Quadro 2. Caracterização das crianças com deficiência auditiva

\begin{tabular}{|c|c|c|c|c|c|c|}
\hline Participantes & Sexo & Idade & Escolaridade & $\begin{array}{c}\text { Grau da perda } \\
\text { auditiva }\end{array}$ & Dispositivo utilizado & $\begin{array}{l}\text { Número de irmãos } \\
\text { residentes na casa }\end{array}$ \\
\hline P1 & Masculino & 10 anos & $5^{\circ}$ ano & Moderada bilateral & AASI bilateral & 1 \\
\hline P2 & Feminino & 11 anos & $5^{\circ}$ ano & Profunda bilateral & $\begin{array}{c}\text { Implante coclear } \\
\text { bilateral }\end{array}$ & 1 \\
\hline P3 & Masculino & 10 anos & $5^{\circ}$ ano & Profunda bilateral & $\begin{array}{c}\text { Implante coclear } \\
\text { bilateral }\end{array}$ & 2 \\
\hline
\end{tabular}

Legenda: $\mathrm{P}=$ Paciente 
terapia. Inicialmente, apresentou-se o projeto, por meio de roda de conversa. Posteriormente, enfatizou-se o objetivo do encontro. Os elementos da ferramenta estavam dispostos em uma mesa na sala e, após a breve explanação, as participantes foram convidadas a se aproximar, manipular o material e a representar, por meio dos elementos disponibilizados, uma situação vivenciada em seu cotidiano, na qual apresentavam alguma dificuldade.

A partir dos ambientes contidos na ferramenta, foram propostos os seguintes cenários: casa, escola (local de reuniões de pais e professores e área onde os pais deixam e buscam seus filhos), shopping e uma área externa. Os personagens e objetos foram confeccionados de forma semelhante aos já existentes. Durante a avaliação, realizou-se anotação dos relatos dos participantes.

\section{RESULTADOS}

As mães avaliaram os cenários e personagens da ferramenta My World, a partir das questões disparadoras sobre os cenários e personagens e foram identificados os seguintes aspectos principais:

1. O que o(a) senhor(a) achou dos cenários e personagens?

- Mães apresentaram comentários positivos:

M1. Explorar locais do cotidiano deve ser o ponto de partida; M2. Ajuda os pais de uma forma lúdica;

M3. Pais precisam aprender a educar seus filhos. Não nascem sabendo.

2. A utilização dos cenários e personagens, de alguma forma, pode ajudar a refletir sobre a relação pais-filho?

- Mães concordaram que sim:

M1 e M2. A casa é o espaço mais íntimo; terão oportunidade de partilhar conhecimentos adquiridos na família;

M3. Ajuda a refletir sobre o que precisa ser mudado, no concreto.

3. O que o(a) senhor(a) mudaria e/ou acrescentaria nesses cenários e personagens?

- Todas sugeriram alguma mudança:

M1. Mudaria o cenário da casa para algo mais próximo do cotidiano da maioria das nossas crianças;

M2. Colocaria brinquedos educativos;

M3. Colocaria um supermercado. É um ambiente em que eles precisam aprender a se comportar.

As análises dos relatos das mães sobre os componentes da ferramenta foram categorizadas de acordo com o cenário, personagens e objetos avaliados, conforme segue.

- Cenário da escola:

M1: Apontou como sendo o "lugar principal" e enfatizou que professores também necessitam de treinamento de habilidades sociais educativas. Emocionou-se ao entrar em contato com este cenário e verbalizou que seu filho havia vivenciado, recentemente, episódio de bullying, relacionado à deficiência auditiva. Ressaltou a importância dos pais serem presentes neste ambiente, criando estratégias para ter acesso a professores, coordenação e direção.

M2: Referiu que "[...] é o ambiente em que os pais mais têm dificuldade de acesso" e salientou a importância de serem trabalhadas estratégias para que os pais consigam o apoio da escola para lidar com a deficiência auditiva.

M3: Ressaltou que é "[...] muito importante a família estabelecer um bom vínculo com a escola" e relatou não ter dificuldades neste aspecto, por ter facilidade de acesso aos professores de sua filha.
Nenhuma mãe sugeriu alterações quanto a este cenário. - Cenário da casa:

M1: Ressaltou não ter problemas de interação em casa. Sugeriu alterações para que a casa representada fosse mais "humilde", no sentido de caracterizar, de forma mais fidedigna, o poder aquisitivo da maior parte da clientela da clínica. Opinou que "[...] cada pai deveria retratar a sua casa por meio de um desenho" e que este ambiente "[...] poderia não ser um cenário predeterminado".

M2: Enfatizou que a casa é um lugar de interação familiar. Referiu "[...] dificuldade em arrumar o ambiente de forma que a filha possa fazer tarefa sem se distrair" e ressaltou acreditar que o uso deste cenário poderia auxiliar a visualizar melhor qual seria o ambiente adequado. Não sugeriu alterações.

M3: Referiu as dificuldades em fazer a criança seguir as regras estabelecidas pelos pais e ressaltou que, muitas vezes, utiliza-se de punição. Acredita que este ambiente poderá “[...] ajudar a entender porque as regras não são cumpridas". Também não sugeriu alterações.

\section{- Cenários externos:}

M1: Verbalizou que não realizaria alterações nestes ambientes e, no momento da avaliação, focou mais na escola e na casa. M2: Ressaltou serem "[...] as áreas mais dificeis de se controlar os comportamentos da criança, por estar em público" e questionou "[...] como reprimir a criança no meio de outras pessoas?". Não apontou alterações nestes ambientes.

M3: Enfatizou a necessidade de que os pais saibam educar seus filhos de modo que eles tenham autonomia em ambientes livres, mas salientou não aceitar que sua filha saia sem a sua supervisão, pois a considera "muito inocente" e questionou "[...] como prepará-la para enfrentar o mundo lá fora?". Sugeriu a criação de mais um ambiente externo (supermercado), por ser um local onde tem muita dificuldade de controlar o comportamento de sua filha.

- Personagens e objetos:

M1: Não sugeriu alterações.

M2: Sugeriu mudar a cor do implante coclear (que está retratado na cor branca) e a inclusão de brinquedos educativos (jogos). M3: Também sugeriu mudar a cor do implante coclear.

\section{DISCUSSÃo}

O presente estudo teve o intuito de contribuir com as práticas centradas na família, ao propor o uso da ferramenta My World com familiares de crianças e adolescentes com deficiência auditiva, com o objetivo de propiciar a aquisição de habilidades que se apliquem ao seu cotidiano. Para isso, procurou-se apresentar os elementos da ferramenta aos familiares de crianças com deficiência auditiva. Assim como em outros estudos realizados com pais, não foi observada a participação da figura paterna ${ }^{(12-15)}$. Mesmo em relação à participação das mães, verificou-se, como uma lacuna do estudo, o número reduzido de participantes. Desta forma, destaca-se a necessidade de se pensar em estratégias que possam favorecer a participação de um número maior de pais e a inclusão da figura paterna.

As práticas de intervenção centradas na família vêm se destacando por contribuírem de forma mais efetiva com o desenvolvimento da criança ${ }^{(1)}$, sendo ressaltada a necessidade de que as intervenções tenham, como ponto de partida, o cotidiano das famílias, de modo a facilitar a incorporação de práticas em seu dia a dia, que contribuam para esse desenvolvimento ${ }^{(2)}$. $\mathrm{Na}$ área da Audiologia, tais práticas vêm sendo evidenciadas por 
propiciarem melhores resultados que a terapia convencional ${ }^{(4,7)}$. No entanto, pesquisam apontaram que ainda existe a necessidade de novas estratégias que aproximem pacientes e familiares de seu cotidiano ${ }^{(3,5)}$. O presente estudo pode ajudar a minimizar esta lacuna e atender o tipo de demanda inerente a estas práticas.

O uso de ferramentas vem se mostrando como possibilidade de intervenção congruente com a proposta preconizada pelas práticas centradas na família, no sentido de possibilitar maior proximidade da criança e sua família com as interações cotidianas ${ }^{(4)}$. Procurou-se, neste estudo, propor o uso dos cenários da ferramenta My World com mães, como uma estratégia que possa contribuir com este processo. Por meio dos relatos das participantes e das respostas ao questionário, observou-se que, de forma geral, os elementos da ferramenta parecem ter contribuído para que as mães identificassem, mais concretamente, dificuldades e facilidades na interação com seu filho com deficiência auditiva, nos diferentes ambientes representados (casa, escola e ambiente externo), o que, por sua vez, pode auxiliar para generalização dos conteúdos trabalhados durante o processo de reabilitação. A partir da análise das participantes, os elementos da ferramenta foram preservados. Assim, em relação ao ambiente de casa, em que M1 sugeriu que cada familiar desenhasse sua própria casa, ressaltou-se que o objetivo era apresentar algo concreto aos pais, para que pudessem se expressar neste ambiente. Procurou-se enfatizar a flexibilidade dos ambientes externos, no sentido de não ser um local fixo. Buscou-se, também, esclarecer que a cor neutra do implante coclear visava a oportunidade dos pais de personalizá-lo, para torná-lo mais semelhante ao da criança, já que costuma ser encontrado em diferentes cores.

Acredita-se que a utilização desses cenários e personagens está alinhada aos princípios norteadores das intervenções centradas na família, como o estabelecimento de suporte emocional às famílias e a promoção da interação pais-criança ${ }^{(8)}$.

\section{CONCLUSÃO}

Considera-se que este estudo pode contribuir para que pacientes e familiares coloquem suas dificuldades e facilidades encontradas em seu dia a dia, em diferentes ambientes, como ressaltado pelas participantes, contribuindo, assim, para maior sucesso no desenvolvimento da linguagem oral de crianças com deficiência auditiva.

A literatura tem colocado que a perda auditiva pode ser uma variável que interfere de forma negativa na interação pais-filhos, sendo enfatizada a necessidade de que os pais de crianças com deficiência auditiva participem de programas com objetivo de desenvolver e/ou aprimorar as práticas parentais consideras positivas. A proposta de utilização de cenários da ferramenta My World com pais de crianças com deficiência auditiva, associada a programas de intervenção que busquem a melhora dessa interação, pode auxiliar no desenvolvimento e/ou aprimoramento de habilidades sociais educativas relacionadas ao cotidiano, o que, por sua vez, pode contribuir com a terapia fonoaudiológica no que se refere ao emprego, em seu dia a dia, das habilidades desenvolvidas durante o contexto terapêutico.

\section{AGRADECIMENTOS}

Ao grupo de fonoaudiólogas que realizou as instruções em português da ferramenta My World: Ms. Paula Paiva, Dra. Marina Morettin Zupelari e Profa. Dra. Deborah Viviane
Ferraz, do Departamento de Fonoaudiologia da Faculdade de Odontologia de Bauru - São Paulo.

\section{REFERÊNCIAS}

1. Dunst CJ. Revisiting "rethinking early intervention". Top Early Child Spec Educ. 2000;20(2):95-104. http://dx.doi.org/10.1177/027112140002000205.

2. Gràcia $\mathrm{M}$, Domeniconi $\mathrm{C}$. Valoración de las rutinas e interacciones comunicativas de familias de preescolares y diseño de una intervención centrada en la família. Investigación Cualitative. 2017;1:116-21.

3. Decker KB, Vallotton CD. Early intervention for children with hearing loss: information parents receive about supporting children's language. J Early Interv. 2016;38(3):151-69. http://dx.doi. org/10.1177/1053815116653448.

4. Espe-Scherwindt M, Serrano AM. Se necesitan 2: el papel de las prácticas centradas en la familia en intervención de la comunicación. Ver. Logop. Foniatr. Audiol. 2016;36(4):162-9.

5. Guijo LM, Delgado-Pinheiro EMC. Caracterização da interação comunicativa entre pais de crianças e adolescentes deficientes auditivos que utilizam comunicação oral. Rev CEFAC. 2016;18(5):1060-8. http://dx.doi.org/10.1590/1982-0216201618523515.

6. Alves AMVS, Lemes VAMP. O poder da audição na construção da linguagem. In: Bevilacqua MC, Moret ALM, organizadores. Deficiência auditiva: conversando com familiares e profissionais de saúde. São José dos Campos: Pulso; 2005. p. 161-78.

7. Bevilacqua MC, Formigoni GMP. O desenvolvimento das habilidades auditivas. In: Bevilacqua MC, Moret ALM, organizadores. Deficiência auditiva: conversando com familiares e profissionais de saúde. São José dos Campos: Pulso; p. 179-201.

8. Moeller MP, Carr G, Seaver L, Stredler-Brown A, Holzinger D. Best practices in family-centered early intervention for children who are deaf or hard of hearing: an international consensus statement. J Deaf Stud Deaf Educ. 2013;18(4):429-45. http://dx.doi.org/10.1093/ deafed/ent034. PMid:24030911.

9. Abramides DVM. Aspectos psicossociais da aquisição e desenvolvimento da linguagem. In: Lamonica DAC, organizador. Estimulação da Linguagem: aspectos teóricos e práticos. São José dos Campos: Pulso; 2008. p. 29-41.

10. Graciano MIG, Lehfeld NAS. Estudo socioeconômico: indicadores e metodologia numa abordagem contemporânea. Serv. Soc. \&. Saúde. 2010;9(9):157-86.

11. Ida Institute [Internet] 2014 [citado em 2014 Nov 20]. Disponível em: http://idainstitute.com

12. Bolsoni-Silva AT. Intervenção em grupo para pais: descrição de procedimento. Temas Psicol. 2007;15(2):217-35.

13. Orti N, Bolsoni-Silva A, Villa M. Assessment of the effects of a parental intervention with mothers of children with internalizing problems. AIR. 2015;4(5):279-92. http://dx.doi.org/10.9734/AIR/2015/16181.

14. Pinheiro MIS, Haase VG, Del Prette A, Amarante CLC, Del Prette ZAP. Treinamento de habilidades sociais e educativas para pais de crianças com problemas de comportamento. Psicol Reflex Crit. 2006;19(3):40714. http://dx.doi.org/10.1590/S0102-79722006000300009.

15. Rocha MMR, Del Prette ZAP, Del Prette A. Avaliação de um programa de habilidades sociais educativas para mães de crianças com TDAH. Acta Comport. 2013;21(3):359-75. 